Experiencia educativa

\title{
Experiencia educativa con Kodu en El Salvador
}

\author{
Educational experience with Kodu in El Salvador \\ Hugo A. Aguirre * \\ hugo.aguirre@edge.com.sv \\ ISSN 1996-1642, Editorial Universidad Don Bosco, año 7, No.12, Julio-Diciembre de 2013, pp.67-75 \\ Recibido: 15 de agosto 2012 Aceptado: 12 de diciembre de 2012
}

Resumen

Esta es la sistematización de la experiencia de usar Kodu, un programa 3-D de diseño de videos para desarrollar habilidades computacionales en los estudiantes de primaria en escuelas de EL Salvador. La experiencia se desarrolló como una competencia en todo el país y entre sus resultados están el desarrollo precisamente de producciones de video y niveles altos de competencias computacionales a través del uso de Kodu.

Palabras clave: competencia

computacional, video, Kodu

\section{Abstract:}

This is the experience of using Kodu, a 3-D video design computing program, to develop computing competences in primary school students in El Salvador. The experience was carried out as a contest at a countrywide level and the outcomes show high levels of development of computing competencies allowed by the use of Kodu in the participants.

Keywords: computing competences, video, Kodu

* Master en Tecnología educativa y subdirector en T-Box El Salvador 


\section{Introducción}

El presente artículo tiene como propósito documentar la experiencia capitalizada en la primera Copa Kodu en El Salvador, explicando las principales etapas y algunos fundamentos pedagógicos con los que se busca aprovechar las tecnologías y su capacidad para contribuir al aprendizaje de los estudiantes.

En octubre de 2011, la gerencia de programas educativos de Microsoft para Centroamérica propuso el reto de organizar esta competencia. La primera pregunta que surgió fue: ¿Qué es Kodu? La respuesta fue sencilla y muy motivante: "Kodu es un lenguaje de programación visual elaborado por Microsoft específicamente para crear juegos de video. Está diseñado para ser accesible a los niños y agradable para todos. Kodu proporciona herramientas fáciles para crear paisajes elaborados en 3D así como para controlar la iluminación y la cámara. La programación Kodu involucra la selección de bloques visuales para una condición "WHEN" (cuando) y una acción "DO" (hacer)."

Luego de la fase de prueba y aprendizaje del programa, se hicieron tangibles todas las ventajas que este podría traer al introducirlo adecuadamente en los centros educativos de El Salvador. Principalmente se identificaron tres destrezas del Siglo XXI que se podían fortalecer en los estudiantes que estuvieran expuestos a la experiencia de diseñar un juego de video propio: la creatividad, el trabajo en equipo y la capacidad de resolver problemas.

\section{Prueba piloto}

Una vez que se tuvo claridad en las ventajas del uso de este programa, se decidió llevar a cabo una primera experiencia piloto con estudiantes interesados en esta área. Con el auspicio de HOLCIM El Salvador, se impartió un curso de verano para estudiantes de centros escolares en la ciudad de Metapán, departamento de Santa Ana. El curso se denominó "Aventura espacial" y fue impartido en los meses de noviembre y diciembre de 2011 por instructores T-Box; tuvo una duración de 40 horas. Los resultados fueron muy interesantes, sobre todo en la motivación que mostraron los participantes en el diseño y programación de sus juegos de video.

"La programación dejó de ser algo aburrido y lleno de código para convertirse en algo divertido de paisajes tridimensionales y objetos animados", resume uno de los instructores de este curso de verano. En este curso participaron aproximadamente 80 estudiantes de sexto y séptimo grado, quienes tuvieron la oportunidad de completar diferentes desafíos que les permitieron explorar las principales herramientas de diseño y las estructuras de programación Kodu. Para cerrar el curso, los estudiantes tuvieron la oportunidad de mostrar, explicar 
y "vender" su juego de video a sus compañeros, padres de familia y demás miembros de la comunidad.

\section{Pensamiento computacional}

El potencial de la computación para innovar y crear nuevos diseños es básicamente ilimitado. Pensadores de la computación son los creadores, diseñadores y desarrolladores de herramientas tecnológicas y de sistemas que actualmente contribuyen en casi todos los campos del quehacer humano. La computación ha permitido grandes avances que van desde investigación científica hasta nuevas formas de comunicación e interacción social.

Según la Sociedad Internacional de Tecnología Educativa (ISTE por sus siglas en inglés) y la Asociación de Docentes en Ciencias de la Computación (CSTA), el Pensamiento Computacional es un proceso de solución de problemas que abarca las siguientes competencias:

1. Formular problemas de manera que permitan usar computadoras y otras herramientas para solucionarlos.

2. Organizar datos de manera lógica y analizarlos.

3. Representar datos mediante abstracciones, como modelos y simulaciones.

4. Automatizar soluciones mediante pensamiento algorítmico (una serie de pasos ordenados).

5. Identificar, analizar e implementar posibles soluciones con el objeto de encontrar la combinación de pasos y recursos más eficiente y efectiva.

6. Generalizar y transferir ese proceso de solución de problemas a una gran diversidad de estos.

Estas competencias se apoyan en una serie de actitudes que son dimensiones esenciales del Pensamiento Computacional. Estas actitudes incluyen:

- Confianza en el manejo de la complejidad

- Persistencia al trabajar con problemas difíciles

- Tolerancia a la ambigüedad

- Habilidad para comunicarse y trabajar con otros para alcanzar una meta o solución común

El Pensamiento Computacional es un enfoque para resolver un problema determinado que facilita la integración de tecnologías digitales con ideas humanas. No sustituye el énfasis en la creatividad, razonamiento o pensamiento crítico; refuerza dichas competencias y posibilita formas de organizar el problema de manera que la computadora pueda ayudar a la solución del mismo. El Pensamiento Computacional permite que los estudiantes alcancen 
mejores resultados de aprendizaje en diferentes asignaturas escolares, ya que fortalecen sus habilidades de pensamiento de orden superior.

Actualmente es difícil encontrar una ocupación en la que las personas y la tecnología no interactúen. Por medio del Pensamiento Computacional los estudiantes pueden comprender cómo, cuándo y dónde las computadoras y otras herramientas digitales pueden ayudarlos a resolver problemas de una manera más eficiente, que además amplía su propio pensamiento. Fomentar el Pensamiento Computacional no significa que todos los estudiantes vayan a trabajar en la ciencia de la computación, ya que su alcance trae impactos positivos en diferentes áreas y ocupaciones.

\section{La Copa Kodu}

Teniendocomometaprincipalelfortalecimiento delPensamientoComputacional, se inició la preparación de la Copa Kodu en El Salvador. En Latinoamérica este certamen únicamente se ha desarrollado en Puerto Rico y Colombia, por lo que había que planificar las diferentes etapas de la competencia y crear todos los materiales necesarios para llevarla a cabo. La Copa Kodu se definió como una competencia en la que se busca reconocer a los estudiantes que demuestren excelencia en el diseño de juegos de video con la plataforma Kodu Game Lab.

En El Salvador, esta iniciativa tiene el respaldo de Microsoft El Salvador, EDGE con su marca T-Box, y de JMTe/com. Es importante destacar en este apartado que la empresa privada y otros actores de la sociedad deben y pueden apoyar propuestas educativas que beneficien el aprendizaje de los estudiantes. La Copa Kodu es un claro ejemplo del trabajo de una red colaborativa, en la cual participan centros educativos públicos y privados, docentes, estudiantes, padres de familia y la empresa privada. Todos los actores motivados por la emoción de participar en un evento nacional, totalmente innovador y con beneficios concretos para los estudiantes. Una lección de esta experiencia es que los centros educativos no deben verse como islas dentro de una comunidad, pueden integrarse con los diferentes actores de la sociedad para crear proyectos que beneficien diferentes áreas de la institución con capacitación, asesoría especializada, tecnología e infraestructura, por ejemplo.

Una vez conseguido el apoyo para llevar a cabo la iniciativa, se definió el calendario de actividades que se seguirían para la edición 2012. La primera fecha fijada fue el lanzamiento de la competencia. En este evento se darían a conocer todos los detalles de la Copa Kodu: requisitos de participación, etapas, reglamento y premios, entre otros. 
Uno acción central previa a la competencia fue la elaboración de las bases de competencia. Se determinaron los requisitos básicos de participación, entre ellos los siguientes:

- La Copa Kodu es una competencia abierta para centros educativos públicos y privados de todo el país.

- Los equipos deben estar formados por 2 estudiantes de un mismo centro educativo. Cada colegio podía participar con un máximo de dos equipos.

- Los estudiantes deben estar inscritos en sexto, séptimo u octavo grado.

- Cada equipo de estudiantes debe estar asesorado por un docente "entrenador".

- Los entrenadores deben participar en una de las jornadas de capacitación de Kodu programadas en el calendario de actividades.

Además de los requisitos de participación, se definieron los lineamientos que se debían seguir en la elaboración de los juegos de video y el proceso para llevar a cabo la evaluación de cada uno de los proyectos presentados.

Otro de los aspectos fundamentales de la etapa de preparación fue construir los criterios e indicadores que se utilizarían para evaluar cada uno de los proyectos propuestos por los equipos participantes. Se tomaron como base los criterios utilizados en la Copa Kodu de Puerto Rico y sobre ellos se definieron los indicadores de evaluación. Los criterios definidos para evaluar cada uno de los juegos de video son:

- Innovación: nivel de creatividad y originalidad del juego.

- Entretenimiento: actividad y emoción que brinda el juego.

- Calidad de producción: gráficos, funcionalidad y temática.

- Presentación: claridad del resumen del juego, precisión de las instrucciones para jugarlo y calidad de la presentación.

Luego de tener definidos todos los detalles de la competencia, se procedió a elaborar el material promocional para dar a conocer la iniciativa. Se diseñaron varios recursos, entre ellos el documento informativo para los centros educativos, el sitio web oficial, afiches y boletines. En esta etapa la idea se había concretizado y era momento de comenzar a ejecutarla.

\section{Lanzamiento e inscripción}

Tal como se programó en el calendario de actividades, el lanzamiento de la Copa Kodu se llevó a cabo el 25 de abril en las oficinas de Microsoft El Salvador. En este evento participaron directores de centros educativos, coordinadores de 
tecnología, docentes y los patrocinadores de la competencia. Aquí se detallaron los requisitos de participación, el calendario de actividades, el reglamento y los premios.

El evento de lanzamiento confirmó lo que habíamos experimentado en la prueba piloto: diseñar un juego de video original es un proyecto retador, interesante y con grandes beneficios para fortalecer destrezas de pensamiento. Directivos escolares y docentes se mostraron muy interesados en participar, dando como resultado que varios centros educativos realizaron eliminatorias internas para definir a sus equipos representantes. El período de inscripción se abrió por un mes, desde la fecha de lanzamiento hasta el 25 de mayo. Un total de 49 equipos de diferentes centros educativos se inscribieron a la Copa Kodu.

\section{Jornadas de capacitación}

Una condición fundamental en la introducción de una innovación educativa es el proceso de formación para docentes. En la Copa Kodu esta condición fue una prioridad y se organizaron jornadas de formación para los docentes que funcionarían como "entrenadores" de sus equipos. Asistir a estas jornadas de capacitación fue un requisito, con lo cual se buscaba garantizar una adecuada introducción de esta nueva tecnología en cada institución.

Se organizaron 3 jornadas de capacitación con grupos distintos de docentes. En cada jornada se contó con un promedio de 15 participantes y la duración del curso fue de 8 horas, es decir, un día completo. Para llevar a cabo este proceso, se contó con la colaboración de 3 instituciones que facilitaron sus instalaciones y se convirtieron en sedes de capacitación: Colegio García Flamenco, Liceo Getsemaní y FUSALMO. Cada jornada fue evaluada por los participantes y se tuvo una retroalimentación muy positiva de los resultados obtenidos.

\section{Pre-evaluación}

Los proyectos que cada equipo de estudiantes preparó con el apoyo de su docente se recibieron entre el 28 de mayo y el 22 de junio. Cada proyecto constaba de dos elementos básicos:

Ben este documento cada equipo colocó los datos generales, características específicas de su juego de video y una descripción del proceso seguido para elaborarlo.

- Juego de video: cada equipo envió el archivo fuente de su juego de video (*.KODU). Al ejecutar este archivo los miembros del jurado podían explorar el juego y revisar todos sus elementos: escenario, objetos, instrucciones y configuraciones. 
El equipo de Investigación y Desarrollo de T-Box estuvo a cargo de realizar esta etapa de pre-evaluación, la cual daría como resultado el listado de los 20 proyectos que clasifican a la final de la competencia. Los criterios para evaluar cada proyecto se definieron en la etapa de preparación y, basado en dichos criterios, se produjo la guía de evaluación con 10 indicadores específicos. El puntaje máximo que podía obtener cada equipo es de 100 puntos, pues cada indicador se ponderó con una escala del 1 al 10.

En la pre-evaluación, los miembros del equipo encargado de esta etapa identificaron que la mayoría de proyectos presentados constaba de una conceptualización original del juego, con un nivel de diseño y programación muy bien logrado. Uno de los evaluadores, lo define así: "Este proceso de diseño del juego de video en Kodu combina muchas de las habilidades requeridas en el Siglo XXI, las cuales son indispensables para el éxito de los estudiantes en un futuro: pensar creativamente, comunicarse, analizar, colaborar, diseñar y aprender continuamente."

Crear proyectos con Kodu también ayuda a los estudiantes a desarrollar fluidez en el manejo de la tecnología. ¿Qué significa la fluidez? Para considerar que una persona domina el español, el inglés o cualquier otro idioma, es necesario que aprenda no sólo a leer sino también a escribir, es decir a expresarse con el lenguaje. De manera similar, tener competencia en tecnología informática implica no solamente interactuar con la computadora, sino además crear y resolver problemas con ella. iNuevamente surge el Pensamiento Computacional!

Muchos estudiantes no elegirán la programación como su profesión, así como la mayoría no serán escritores profesionales. Pero aprender a programar trae beneficios muy concretos: permite que los estudiantes se expresen de manera más completa y creativa, les ayuda a desarrollarse como pensadores lógicos y a entender el funcionamiento de las nuevas tecnologías que encuentran las diferentes actividades de su vida.

\section{Finalistas}

"El juego de video trata de ganarle una carrera a tu oponente y con seguir 50 puntos. El circuito o la pista donde corren está dividida en seis mundos: el primer mundo está sumergido en agua, el segundo es un desierto, el tercero es una isla de nieve, el cuarto es un bosque, el quinto es un laberinto y la isla final es en la que está ubicada la fábrica." Así describen su juego los estudiantes del Colegio Bautista San Salvador, uno de los proyectos seleccionados como finalistas en la etapa de pre-evaluación. El título de este juego es "El Gran Prix de la Mega Fábrica". 
Del total de proyectos recibidos, la etapa de pre-evaluación dio como resultado un listado de 20 finalistas. Estos finalistas son convocados a la gran final del certamen, la cual se programó para el sábado 18 de agosto. En este evento los estudiantes deben dar a conocer su juego a todos los visitantes y al jurado calificador, explicando el proceso seguido para crearlo y demostrando cómo funciona.

La evaluación que realizó el jurado calificador de la final estuvo basada en los mismos criterios e indicadores empleados en la pre-evaluación. Cada equipo debió preparar su stand de exposición desde el cual podrá "vender" su juego de video a los asistentes y también tuvo un tiempo determinado para explicar su proyecto a los jueces. Al final de la jornada, se premió a los tres equipos que obtuvieron las mejores evaluaciones.

\section{Algunos aprendizajes de la experiencia}

Todo el proceso ha estado lleno de mucho entusiasmo y aprendizaje. Dentro de estos aprendizajes se pueden destacar:

Esta competencia motivó a muchos docentes y estudiantes de centros educativos públicos y privados del país a introducirse al área del diseño y la programación de juegos de video, trayendo como principal beneficio el desarrollo y fortalecimiento del Pensamiento Computacional.

Los centros educativos están muy interesados en participar en iniciativas que promuevan innovaciones dentro del proceso de enseñanza y aprendizaje. Estas innovaciones pueden surgir desde diferentes actores, incluyendo las mismas instituciones educativas en coordinación con la empresa privada, gobierno, fundaciones y otro tipo de organizaciones interesadas en fomentar la calidad educativa.

Toda innovación educativa que se desee introducir en una institución debería estar acompañada de un plan de implementación que incluya las principales variables que garanticen su éxito. Una nueva tecnología, por ejemplo, puede ser muy buena, pero no tendrá los resultados esperados sino se complementa con un proceso de capacitación, acompañamiento y evaluación constante. Hay que fomentar las innovaciones educativas, pero hay que introducirlas adecuadamente para que brinden el impacto buscado.

iEl pensamiento computacional es clave para los estudiantes! Los cuestionamientos acerca de la utilidad de la clase de computación quedan resueltos con varias investigaciones que apoyan la necesidad de desarrollar en los estudiantes destrezas tecnológicas que les ayuden a ampliar su capacidad 
para resolver problemas. El reto está, entonces, en aprovechar al máximo la clase de informática impartiendo metodologías y contenidos que promuevan el desarrollo del pensamiento computacional.

Los resultados de esta experiencia educativa son muy positivos, lo cual permite pensar que esta primera edición es solo el inicio de un certamen que estará vigente por varios años para el beneficio de la comunidad educativa del país. Los estudiantes están dispuestos a asumir retos, así que lo menos que se puede hacer es proponerles alternativas de provecho para su formación personal y académica. iQué siga la innovación educativa!

\section{Referencias}

Sociedad Internacional para las Tecnologías en Educación (ISTE) y la Asociación de Maestros de Ciencia de la Computación (CSTA). (201 1). Pensamiento Computacional. Recuperado el 20 de Julio de 2012, de International Society for Technology in Education: http://www.iste.org/

Technology Box Inc. (25 de abril de 2012). Copa Kodu El Salvador. Recuperado el 20 de julio de 2012, de http://www.tboxplanet.com/web/kodu2012 
